\title{
Wavenumber Spectrum in the Gulf Stream from Shipboard ADCP Observations and Comparison with Altimetry Measurements
}

\author{
Dong-Ping Wang AND Charles N. Flagg \\ School of Marine and Atmospheric Sciences, Stony Brook University, \\ Stony Brook, New York
}

Kathleen Donohue AND H. Thomas Rossby

Graduate School of Oceanography, University of Rhode Island, Kingston, Rhode Island

Corresponding author address: Dong-Ping Wang, SOMAS, Stony Brook University, Stony Brook, NY 11794, U.S.A.

E-mail: dong-ping.wang@stonybrook.edu. 


\begin{abstract}
The wavenumber spectra for velocity and temperature in the Gulf Stream region are calculated from a decade (1994-2004) of shipboard acoustic Doppler current profiler (ADCP) measurements taken as part of the Oleander Project. The velocity and temperature spectra have comparable magnitude, in terms of the kinetic and potential energy, and both indicate a $k^{-3}$ slope. These results are in agreement with twodimensional quasigeostrophic turbulence theory. In contrast, velocity spectrum determined from satellite altimetry sea surface height yields a significantly higher energy and a $k^{-2}$ slope. This discrepancy between directly-measured and altimeter-derived velocity spectra suggests a noise contribution to altimetry along-track sea surface height data. These results also highlight large gaps in the current understanding of the nature of surface geostrophic turbulence.
\end{abstract}




\section{Introduction}

Mesoscale (10-100 km) eddies are dominant motions in the upper ocean. They arise primarily from baroclinic instability and scale to the baroclinic deformation radius $(\sim 30$ $\mathrm{km}$ in mid-latitudes). Energy is transferred through nonlinear turbulence interactions and is ultimately dissipated by viscosity. A fundamental question is how the equilibrium energy spectrum is established. In three-dimensional homogeneous isotropic turbulence, energy cascades to smaller scales following the Kolomogroff $k^{-5 / 3}$ law. In twodimensional turbulence, due to energy and enstrophy (mean-square vorticity) constraints, energy is transferred to scales larger than the scale where energy is inserted ('inverse cascade') while enstrophy is transferred in the opposite direction to the smaller scales ('enstrophy cascade') (Kraichnan, 1967). A similarity argument leads to a $k^{-5 / 3}$ law for the inverse cascade and a $k^{-3}$ law for the enstrophy cascade. Charney (1971) in his seminal geostrophic turbulence study showed that the two-dimensional turbulence theory also holds for stratified quasigeostrophic (QG) flows. Hence, the direct cascade to smaller scales is also expected to follow a $k^{-3}$ law. Charney's argument also leads to an equipartition between kinetic and potential energy.

The QG turbulence theory has been verified for the atmospheric synoptic scales using wavenumber spectra determined from the global reanalyses and direct aircraft measurements (Nastrom and Gage, 1985). In the atmosphere, the baroclinic deformation radius is $\sim 1000 \mathrm{~km}$, and the dominant scales are in the range of $1000-3000 \mathrm{~km}$. The oceanographic observations, on the other hand, generally lack adequate spatial and temporal coverage, and velocity wavenumber spectra have been derived only indirectly from altimetric sea surface height (SSH) measurements. Stammer (1997), using the first 3 
years of data from the TOPEX/Poseidon (T/P) mission, obtained a global SSH spectrum of $k^{-4.6}$. The corresponding surface slope (geostrophic velocity) spectrum of $k^{-2.6}$ appears to be consistent with the $k^{-3}$ QG turbulence. However, the SSH spectrum in the Gulf Stream region is closer to $k^{-4}$ and the corresponding velocity spectrum $k^{-2}$. Le Traon et al. (2008), using all available altimeter measurements (T/P, Jason-1, Geosat Follow-On, and Envisat) in a 4 year period (2002-2005), showed that all oceanic high eddy activity areas have the same $k^{-4}\left(\sim k^{-11 / 3}\right)$ slope. The inferred $k^{-2}\left(\sim k^{-5 / 3}\right)$ velocity spectrum is significantly different from the QG spectrum. The $k^{-2}$ velocity spectrum, on the other hand, is consistent with the surface quasigeostrophic theory (SQG) which assumes conservation of surface temperature variance (Blumen, 1978; Held et al, 1995). Recent submesoscale eddy-resolving numerical model experiments also found a $k^{-2}$ velocity spectrum at the surface (Lapeyre and Klein, 2006; Klein et al 2008; Capet et al 2008a-c). While the evidence for SQG is strong, the conclusion of Le Traon et al. (2008) nevertheless is tentative. This is because the altimetric velocity estimate can be significantly impacted by noise in surface height measurements.

In this note, we take advantage of the long-term $(1994$ - 2004) nearsurface velocity and temperature observations from the Oleander Project (http://www.po.gso.uri.edu/rafos/research/ole/index.html) to compute the wavenumber spectra in the Gulf Stream region. The directly measured wavenumber spectrum also is compared with the altimetric velocity spectrum. The implication on surface geostrophic turbulence is discussed.

\section{Analysis}


In this study, 10 years (1994 - 2004) of shipboard acoustic Doppler current profiler (ADCP) observations from the Oleander Project are used to calculate the wavenumber spectra for nearsurface velocity and temperature in the Gulf Stream region. From 1994 through 2004, a $150 \mathrm{kHz}$ RD Instruments narrow-band ADCP, mounted on the M/V Oleander measured upper-ocean velocity during the container ship's weekly round-trip between New York Harbor and Bermuda. Data are averaged every 5 minutes, resulting in a horizontal resolution of approximately $2.4 \mathrm{~km}$. In the vertical, the bin size ranges between 8 and $16 \mathrm{~m}$ in the open ocean. The sea surface temperature is measured at the instrument level, about $5 \mathrm{~m}$ below the surface.

The Oleander observations have previously been used to study interannual variations of the Gulf Stream surface transport (Rossby et al., 2005), seasonal variations of the shelf/slope jet in Middle Atlantic Bight (Flagg et al., 2006), and Gulf Stream warm-core rings (Wei et al., 2008). In this study we focus on the open ocean to be compatible with altimetry measurements. We use only transects of at least $90 \%$ good observations to assure high data quality. For each $\sim 1,000 \mathrm{~km}$ long transect, the data are interpolated onto a $2 \mathrm{~km}$ grid using a Barnes filter (Daley, 1991) with a correlation length of $2 \mathrm{~km}$ (The filter has no impact on scales $>10 \mathrm{~km}$ ); if the data gap is too large to allow for meaningful interpolation, the transect is discarded. This strict screening process results in a total of 213 transects (out of $>700$ potential transects) spread over the 10-year period. Mean current and velocity ellipses along the Oleander track are shown in Figure 1. They are similar to those found in Rossby et al (2005). Near the Gulf Stream axis the ellipses are polarized in the downstream (zonal) direction, reflecting the effects of Gulf Steam 
meanders, while away from the Gulf Stream, the ellipses indicate a more isotropic eddy field.

To calculate the wavenumber spectrum, the time (ensemble) mean is first removed from each transect, in order to be compatible with the altimetry which does not measure the mean sea surface height. Including the mean velocity profile slightly increases the energy level at large scales but has no effect on the spectral shape. For each transect (of velocity and temperature), the spatial mean and linear trend are removed, the residuals are multiplied by a cosine bell (Hanning) window, and a fast Fourier Transform (FFT) is applied. The spectrum is the sum of the squares of the Fourier components at each wavelength averaged over all transects. There are about 240 wavenumber estimates at a resolution of $\sim 10^{-3}$ cycle per $\mathrm{km}$ ( $\left.\mathrm{cpkm}\right)$. To determine the number of degrees of freedom (DOF), we use a conservative estimate counting only transects that were taken at least two weeks apart. This leads to about 240 DOF. We also assume the speed of the vessel (16 knots) is much greater than the speed of along-track movement of disturbances.

The temperature spectrum is converted into potential energy spectrum as follows. In QG theory, the potential energy (ignoring the $1 / 2$ factor) is given by:

$$
P E=\frac{1}{N^{2}}<b^{2}>\approx \alpha<T^{2}>
$$

where $\mathrm{N}^{2}$ is the Brunt-Vaisala frequency, $b$ is the buoyancy $\left(=g \rho^{\prime} / \rho_{o}\right)$, and $\alpha$ is an empirical constant relating temperature and buoyancy. Because the density change is affected by both temperature and salinity, we need to assume that the $\mathrm{T} / \mathrm{S}$ relation is not affected by eddy stirring. (Seasonal heating is largely eliminated as a result of removing the spatial mean of each transect in the spectrum calculation.) In the Gulf Stream, mean temperature changes (in the vertical) by about $4^{\circ} \mathrm{C}$ over the upper $100 \mathrm{~m}$, and density by 
about $2 \mathrm{~kg} \mathrm{~m}^{-3}$. If we use this ratio, which takes into consideration the salinity contribution, $\alpha$ is about 0.1 (in MKS unit). While this estimate is crude it is sufficient for this study since buoyancy is expected to mainly depend on the temperature.

Figure 2 shows the estimated zonal and meridional velocity spectra plus the kinetic and potential energy spectra. The velocity spectra are calculated from ADCP measurements $30 \mathrm{~m}$ below the surface; the spectra are essentially identical for velocities at $60 \mathrm{~m}$ or $90 \mathrm{~m}$. Two features stand out in the velocity spectra. First, the velocity component spectra are comparable; the zonal velocity is slightly larger at scales $>100$ $\mathrm{km}$. Second, the velocity spectra show a distinct linear slope of -3 over the entire mesoscale range, $O(10-100 \mathrm{~km})$. In the potential energy (temperature) spectrum, the same -3 slope also seems to hold, though there is an offset of energy level at about $50 \mathrm{~km}$ wavelength. Alternatively, if the slope of the potential energy spectrum is fitted over the entire meso-scale range, the slope would be about -2.3 . Perhaps most interestingly, the kinetic energy and potential energy spectra are remarkably similar over the entire wavenumber range (considering the uncertainty in potential energy estimate). Taken together, three results emerge: a $k^{-3}$ law for velocity spectrum, isotropy of horizontal velocity, and an equipartition between kinetic and potential energy. This is in good agreement with two-dimensional QG turbulence theory.

The $k^{-3}$ slope obtained from ADCP observations does not agree with the $k^{-2}$ slope derived from altimetry measurements cited earlier. To make a careful comparison relevant for the Oleander data set, we looked at the two closest T/P tracks (cycles 50 and 126) for the 10-year T/P repeat-orbit period (1992-2002) over the same latitudinal extent between 32 and $39^{\circ} \mathrm{N}$ (Fig. 1). Because the surface slope estimate is sensitive to spatial 
interpolation (filtering) only tracks with no missing data are included in the analysis, which results in a total of 162 tracks (out of $\sim 700$ tracks). Not counting the T/P tracks taken consecutively ( $\sim 10$ day apart), this results in about 280 DOF. The SSH and geostrophic velocity spectra are calculated following the same procedure as for the Oleander data. Also, because the altimetry along-track resolution is coarser, $\sim 6.2 \mathrm{~km}$, three adjacent Oleander velocity samples are averaged to produce an equivalent $6 \mathrm{~km}$ resolution. The spectrum from the resampled Oleander data though is essentially the same as from the original 2-km data.

Figure 3 shows $\mathrm{SSH}$ and satellite-derived geostrophic velocity spectra; the latter is superimposed over Oleander kinetic-energy spectrum. The SSH spectrum shows a $k^{-4}$ slope and the corresponding geostrophic velocity a $k^{-2}$ slope. These agree with Stammer (1997) and Le Traon et al (2008). The high-wavenumber $(<70 \mathrm{~km})$ tail in the SSH spectrum is due to measurement noise which has strong impact on the velocity spectrum. However, even in the 'signal' range between 70 and $200 \mathrm{~km}$, the energy level of the altimetric velocity spectrum is considerably higher, by about $50 \%$, than that of the Oleander. This suggests that the altimetric velocity spectrum probably is contaminated over a broad range. The $k^{-2}$ slope derived from the altimetric velocity spectrum also appears to be an artifact. The total eddy kinetic energies summed over all wavelengths $>$ $70 \mathrm{~km}$ are 0.136 and $0.178 \mathrm{~m}^{2} \mathrm{~s}^{-2}$ for the Oleander and altimetry respectively.

The Oleander spectrum is calculated from a small subset of 213 transects. Relaxing the data screening to $80 \%$ good, the total number of useful transects is doubled (461). The velocity and temperature spectra from this much larger dataset are indistinguishable from the original spectra. We also note that the Oleander and altimetry spectra are not 
calculated for the same time intervals. To test if this may introduce bias, we re-compute the spectrum using only the Oleander transects taken within 2 days of the satellite pass. To have adequate sample size, we use the larger ( $80 \%$ good) Oleander dataset and the filtered altimetry time series. This results in a total of 121 overlapping records. The spatial filter impacts the altimetry spectrum for shorter wavelengths. However, for wavelength $>80 \mathrm{~km}$, the spectral shapes are essentially the same as in the original spectra.

\section{Discussion}

The apparent good agreement between the Oleander results and QG turbulence theory is surprising. In QG theory, temperature variations are supposed to be located at the thermocline (the first baroclinic mode). In the Gulf Stream, large temperature variances are concentrated in the upper ocean, associated with the movement of fronts, filaments and eddies. The SQG theory which predicts equivalence between surface buoyancy and kinetic energy, in fact, seems to be more consistent with the observation. However, the $k$ $5 / 3$ spectrum predicted by the SQG theory differs significantly from the observed $k^{-3}$ spectrum. How a $k^{-3}$ law remains valid despite large surface temperature variance requires further theoretical investigation. We note, for example, the semi-geostrophic theory, which perhaps is more appropriate to describe Gulf Stream frontogenesis, predicts a $k^{-8 / 3}$ spectrum, very close to $k^{-3}$ (Andrews and Hoskins, 1978). Also, it is not clear if the $k^{-3}$ kinetic energy spectrum in the Gulf Stream is universal. It would be very useful if the present results can be verified for other major current systems like the Kuroshio and the Antarctic Circumpolar Current. 
Neither the magnitude nor the shape of altimetric velocity spectrum agrees with the direct-velocity observations. The assumption that the altimetric velocity spectrum is not affected by the SSH noise in wavelengths $>70 \mathrm{~km}$ is perhaps too optimistic. How the SSH noise should be filtered, which may vary from region to region, is a challenging question. Interpretation of altimetric wavenumber spectrum in the global context should also be treated with caution.

\section{Acknowledgement}

The Oleander Project is supported by NSF grant OCE-0825845 to URI, and OCE0825418 and OCE-0425413 to Stony Brook. We would also like to acknowledge the logistic and processing help of George Schwartze, Sandra Fontana, and the crew of the Oleander and Bermuda Container Line for their crucial efforts and support. 


\section{REFERENCES}

Andrews, D.G., and B.J. Hoskins, 1978: Energy spectra predicted by semi-geostrophic theories of frontogenesis. J. Atmos. Sci., 35, 509-512.

Blumen, W., 1978: Uniform potential vorticity flow. Part I: Theory of wave interactions and two-dimensional turbulence. J. Atmos. Sci., 35, 774-783.

Capet, X., J. C. McWilliams, M. J. Molemaker, and A. F. Shchepetkin, 2008: Mesoscale to submesoscale transition in the California Current System. Part I: Flow structure, eddy flux, and observational tests. J. Phys. Oceanogr., 38, 29-43.

,,--- , and,$- 2008 \mathrm{~b}$ : Mesoscale to submesoscale transition in the California Current System. Part II: Frontal processes. J. Phys. Oceanogr., 38, 44-64.

,,--- , and,$- 2008 \mathrm{c}$ : Mesoscale to submesoscale transition in the California Current System. Part III: Energy balance and flux. J. Phys. Oceanogr., 38, $2256-2269$.

Charney, J., 1971: Geostrophic turbulence. J. Atmos. Sci., 28, 1087-1095.

Daley, R., 1991: Atmospheric Data Analysis, 457 pp., Cambridge Univ. Press, New York.

Flagg, C. N., M. Dunn, D.-P. Wang, H. T. Rossby, and R. L. Benway, 2006: A study of the currents of the outer shelf and upper slope from a decade of shipboard ADCP observations in the Middle Atlantic Bight, J. Geophys. Res., 111, doi:10.1029/2005JC003116.

Held, I. M., R. T. Pierrehumbert, S. T. Garner, and K. L. Swanson, 1995: Surface quasigeostrophic dynamics. J. Fluid Mech., 282, 1-20. 
Klein, P., B. L. Hua, G. Lapeyre, X. Capet, S. Le Gentil, and H. Sasaki, 2008: Upper ocean turbulence from high-resolution 3D simulations. J. Phys. Oceanogr., 38, 17481763.

Kraichnan, R., 1967: Inertial ranges in two-dimensional turbulence. Physics Fluids, 10, 1417-1423.

Lapeyre, G., and P. Klein, 2006: Dynamics of the upper oceanic layers in terms of surface quasigeostrophy theory. J. Phys. Oceanogr., 36, 165-176.

Le Traon, P. Y., P. Klein, B. L. Hua, and G. Dibarboure, 2008: Do altimeter wavenumber spectra agree with interior or surface quasigeostrophic theory? J. Phys. Oceanogr., 38, $1137-1142$.

Nastrom, G.D., and K.S. Gage, 1985: A climatology of atmospheric wavenumber spectra observed by commercial aircraft. J. Atmos. Sci., 42, 950-960.

Rossby, T, C. N. Flagg and K. Donohue, 2005: Interannual variations in upper ocean transport by the Gulf Stream and adjacent waters between New Jersey and Bermuda. J. Mar. Res., 63, 203-226.

Stammer, D., 1997: Global characteristics of ocean variability estimated from regional TOPEX/POSEIDON altimeter measurements. J. Phys. Oceanogr., 27, 1743-1769.

Wei, J., D.-P. Wang, and C.N. Flagg, 2008: Mapping Gulf Stream warm core rings from shipboard ADCP transects of the Oleander Project. J. Geophys. Res., 113, doi:10.1029/2007JC004694. 


\section{Figure Captions}

Figure 1. Mean current and velocity ellipses from Oleander ADCP measurements at $30 \mathrm{~m}$ depth (plotted every $20 \mathrm{~km}$ ). The depth contours (solid lines) and T/P tracks are marked (dash-dotted lines).

Figure 2. (Left) Zonal (solid) and meridional (dash-dotted) velocity spectra, and (right) potential energy (solid) and kinetic energy (dash-dotted) spectra, from the Oleander observations. Dashed lines indicate a -3 slope. The $95 \%$ confidence interval is marked.

Figure 3. (Left) Sea surface height and (right) geostrophic velocity spectra from altimetry measurements, superimposed with kinetic energy spectrum (dash-dotted) from Oleander observations. Dashed lines indicate respectively a $-4,-3$, and -2 slope. The 95\% confidence interval is marked. 


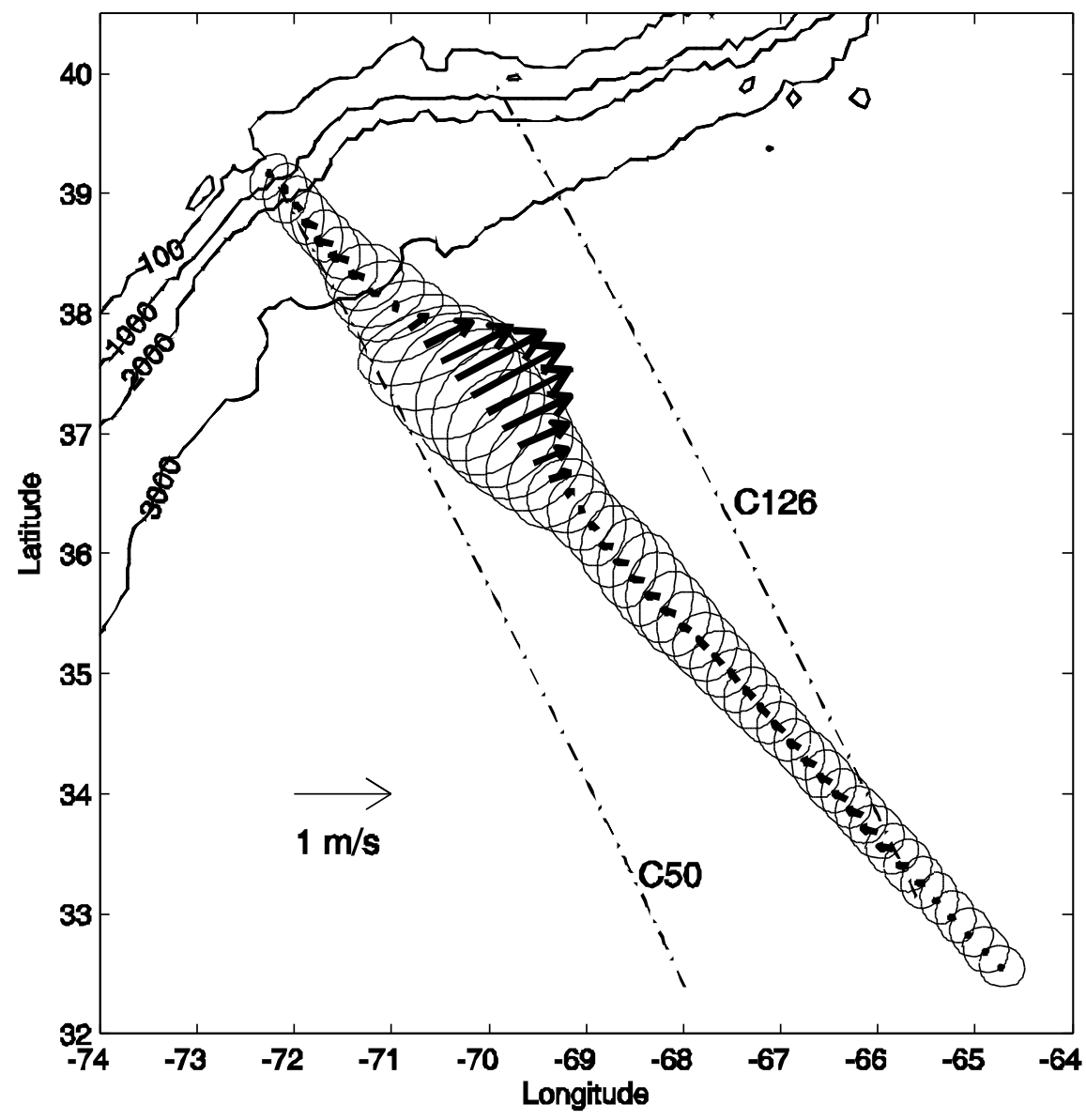

Figure 1. Mean current and velocity ellipses from Oleander ADCP measurements at $30 \mathrm{~m}$ depth (plotted every $20 \mathrm{~km}$ ). The depth contours (solid) and T/P tracks are marked (dashdotted). 

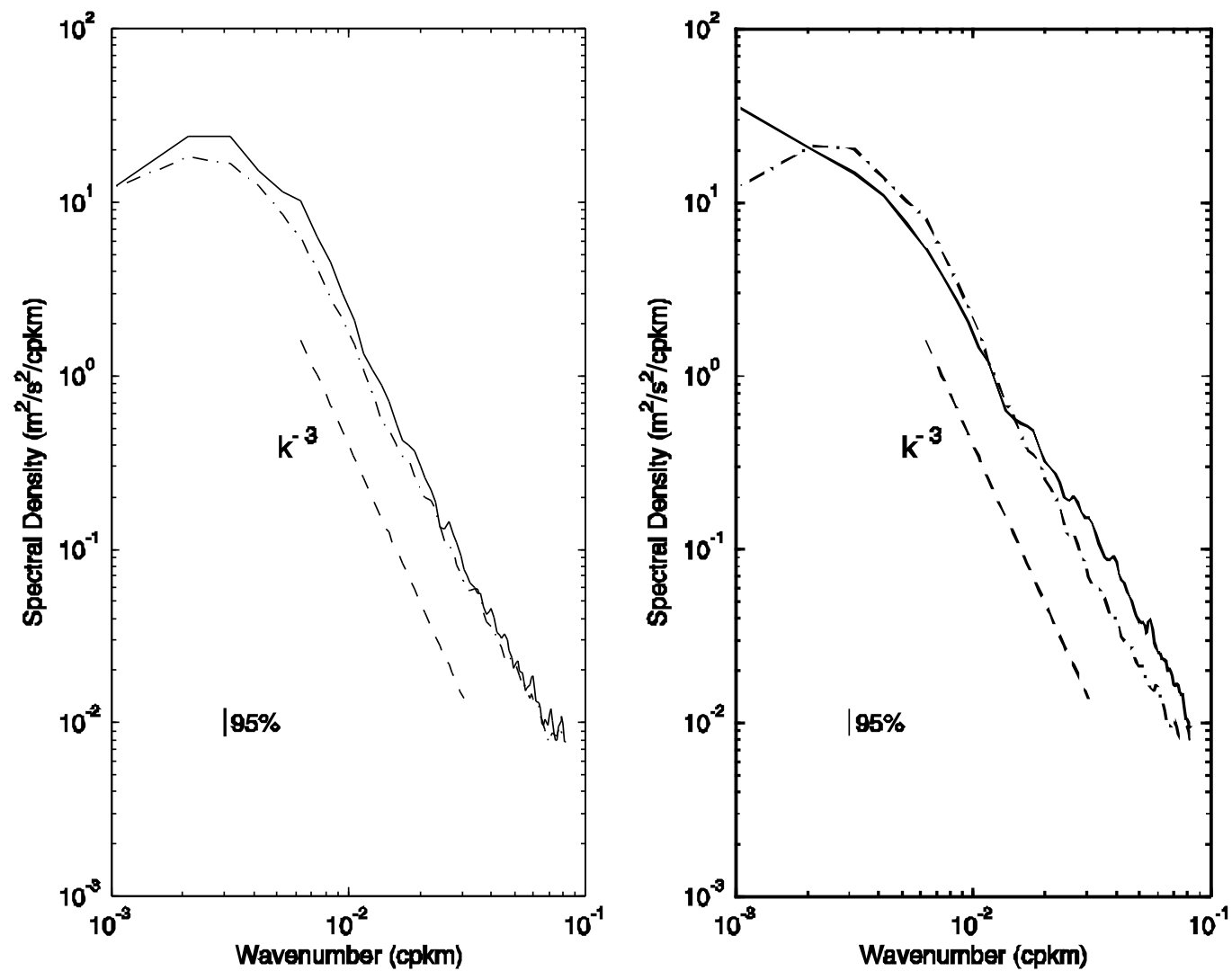

Figure 2. (Left) Zonal (solid) and meridional (dash-dotted) velocity spectra, and (right) potential energy (solid) and kinetic energy (dash-dotted) spectra, from the Oleander observations. Dashed lines indicate a -3 slope. The 95\% confidence interval is marked. 

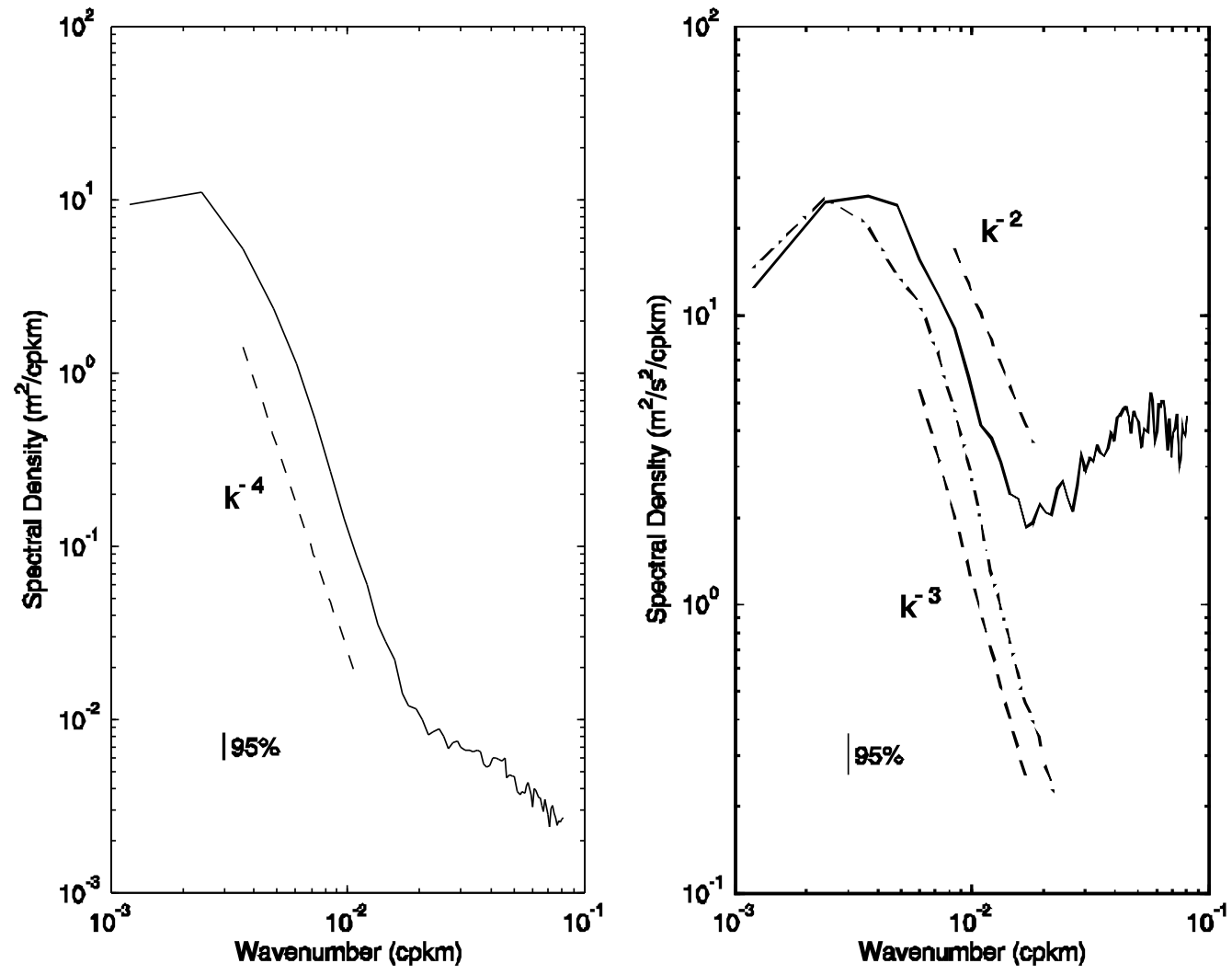

Figure 3. (Left) Sea surface height and (right) geostrophic velocity spectra from altimetry measurements, superimposed with kinetic energy spectrum (dash-dotted) from Oleander observations. Dashed lines indicate respectively a $-4,-3$, and -2 slope. The $95 \%$ confidence interval is marked. 